\title{
Usefulness of an accelerometer-based navigation system in bilateral one-stage total knee arthroplasty
}

Artit Laoruengthana', Piti Rattanaprichavej ${ }^{1 *}$, Thanawat Tantimethanon ${ }^{1}$, Watcharapong Eiamjumras ${ }^{1}$, Passakorn Teekaweerakit ${ }^{1}$ and Krit Pongpirul ${ }^{2,3}$

\begin{abstract}
Background: Bilateral one-stage total knee arthroplasty (BTKA) have increased because it provides a number of advantages. Recently, Accelerometer-based navigation (ABN) system which guide the cutting plane without intramedullary disturbance might result in less endothelial and microvascular damage. Therefore, we hypothesized that the ABN may reduce blood loss, reduce postoperative pain, and better restore BTKA alignment compared to conventional instruments.
\end{abstract}

Methods: We retrospectively compared 44 consecutive patients receiving ABN assisted BTKA (BBTKA) to 57 patients with conventional instruments (CBTKA). Identical pre- and post-operative care was utilized to all patients. The outcome measures assessed were hemoglobin $(\mathrm{Hb})$, calculated blood loss (CBL), blood transfusion, VAS score for pain, morphine consumption, knee flexion angle, and length of stay (LOS). Radiographic assessment included mechanical axis (MA) and component positioning at 3-6 months of follow up.

Results: Both iBTKA and cTKA groups had equivalent demographic data. Postoperative Hb of the cBTKA group was significantly lower than those in the iBTKA group at $24 \mathrm{~h}(p=0.02)$, but there was no significant difference in drain volume, $\mathrm{CBL}$, and blood transfusion rate. For radiographic measures, the iBTKA group had more accurate MA and component orientation, and had a lower number of outliers than those in the cBTKA group $(p \leq 0.01)$, except for the sagittal femoral component angle.

Conclusion: The ABN assisted BTKA could not reduce blood loss or postoperative pain more than CBTKA, nor improve functional recovery. However, the ABN significantly improved the accuracy of MA and prostheses positioning.

Trial registration: The protocol of this study was registered in the Thai Clinical Trials Registry database No. TCTR2 0180731001 on 25 July 2018.

Keywords: Accelerometer-based navigation, Bilateral total knee arthroplasty, Blood loss, Postoperative pain, Mechanical axis

\footnotetext{
* Correspondence: pt-rp@hotmail.com

'Department of Orthopaedics, Faculty of Medicine, Naresuan University, 99

Moo 9 Thapho, Phitsanulok 65000, Thailand

Full list of author information is available at the end of the article
}

(c) The Author(s). 2021 Open Access This article is licensed under a Creative Commons Attribution 4.0 International License, which permits use, sharing, adaptation, distribution and reproduction in any medium or format, as long as you give appropriate credit to the original author(s) and the source, provide a link to the Creative Commons licence, and indicate if changes were made. The images or other third party material in this article are included in the article's Creative Commons licence, unless indicated otherwise in a credit line to the material. If material is not included in the article's Creative Commons licence and your intended use is not permitted by statutory regulation or exceeds the permitted use, you will need to obtain permission directly from the copyright holder. To view a copy of this licence, visit http://creativecommons.org/licenses/by/4.0/. The Creative Commons Public Domain Dedication waiver (http://creativecommons.org/publicdomain/zero/1.0/) applies to the data made available in this article, unless otherwise stated in a credit line to the data. 


\section{Background}

Currently, bilateral one-stage total knee arthroplasty (BTKA) procedures have increased as an alternative option for patients with bilateral involvement [1]. However, there are potential disadvantages concerning BTKA [2], one of which is that it is associated with greater blood loss than the unilateral total knee arthroplasty (UTKA). This significant amount of blood loss may increase intra-articular pressure and swelling of soft tissue which can increase postoperative pain and require higher muscle strength to perform postoperative rehabilitation $[3,4]$. Recent published studies showed approximately 1 1 of calculated blood loss (CBL) and approximately 30\% of allogeneic blood transfusion rate after BTKA, even if tranexamic acid (TXA) was administered [5]. Currently, meta-analysis evaluation of the effects of CAS on postTKA bleeding demonstrated that CAS could be effective in reducing CBL by $185.4 \mathrm{ml}$ as compared to conventional TKA [6]. Thus, emerging technologies that minimize medullary cavity disturbance may help mitigating these potential drawbacks of BTKA [7].

Recently, Accelerometer-Based Navigation (ABN) systems; handheld navigation systems, combine the alignment accuracy of large computer console systems, and ease of use with 2 wireless pods attached to the conventional instruments. The ABN detects the rotatory center location through a stop-and-go movement, and, following a series of short calibration steps, these gyroscopic pods display alignment information and guide the cutting plane within the operative field [8]. Goh et al. [9] demonstrated that $\mathrm{ABN}$ has a comparable number of outliers for the mechanical axis (MA) and coronal prostheses orientation, as compared to non CT-based CAS. In addition to improving limb alignment, another possible benefit using ABN in BTKA is that it may cause less endothelial and microvascular damage by avoiding intramedullary disturbance, and may result in less postoperative hemarthrosis and blood loss, less inflammation, lower postoperative pain intensity, and reduced potential risks related to marrow embolism [7, 10, 11]. Kawaguchi et al. [12] retrospectively reviewed 64 patients who underwent conventional unilateral TKA (32 patients) and ABN assisted TKA (32 patients). They found that the patients in the ABN group had significantly less estimated blood loss when compared to patients using conventional instruments $(422.5 \pm 187.5$ vs $563.6 \pm 261.9 ; p<0.01)$.

Hence, we hypothesized that using ABN would improve the accuracy of postoperative MA and component positioning, and may solve some concerning issues related to conventional BTKA. Because of limited studies for $\mathrm{ABN}$ in BTKA, we conducted the present study to determine whether $\mathrm{ABN}$ can 1) reduce blood loss, 2) reduce postoperative pain, 3) better restore mechanical and prosthesis alignment, and 4) reduce complications after BTKA when compared to conventional system.

\section{Methods}

This investigation was a retrospective, cohort study of patients who had undergone BTKA with either conventional instruments (cBTKA) or accelerometer-based navigation (iBTKA) by a single, senior arthroplasty surgeon (AL) who expertise in both conventional and iASSIST $^{\mathrm{TM}}$ system. All patients with a diagnosis of primary osteoarthritis of both knees and who were undergoing bilateral TKA in the same anesthesia were enrolled. Exclusion criteria included patients with prior knee surgery history, prior knee infection, and hip pathology that severely limited range of motion. Forty-four consecutive patients who received iBTKA during 2018-2019 and a cohort of 57 patients that underwent cBTKA during 2016-2018 were compared. The study was approved by the Institutional Review Board, and informed consent was elicited from every patient.

Regional anesthesia comprising Bupivacaine $(0.5 \%$ Marcaine, AstraZeneca, Sweden), prophylactic antibiotic injection, and tourniquet control at $250 \mathrm{mmHg}$, were applied to all patients. All the surgical procedures were performed by a single surgeon via standard medial parapatellar arthrotomy. For the cBTKA group, the proximal tibial bone was first cut using an extramedullary alignment guide which was targeting perpendicularly to the tibial coronal axis. The distal femoral cut was then done with intramedullary reference guides aiming for $5^{\circ}$ of the valgus in coronal plane, and aiming perpendicularly to the anatomical axis in sagittal plane. Femoral entry point was altered in coronal plane according to the anatomical axis as preoperatively template, while the entry point in sagittal plane was set at $1 \mathrm{~cm}$ anterior to the top of intercondylar notch which allow the intramedullary guide aligned to sagittal femoral axis [13]. The posterior condylar axis was used as a reference for $3^{\circ}$ external rotation of the femoral component. Likewise, the iBTKA group received identical anesthetic and surgical procedures except that the ABN system (iASSIST $^{\mathrm{\tau м}}$, Zimmer inc., Warsaw, IN, USA) was applied to guide proximal tibial and distal femoral bone cuts. For the iBTKA group, the femoral spike was impacted at the similar entry point of the distal femur, and the reference pod was attached to the spike. The leg was then moved around the hip center to orientate the MA of the femur, and the distal femoral cut was made perpendicularly to the mechanical alignment in coronal plane and $1^{\circ}$ of flexion from the MA in sagittal plane. For femoral rotation, the anterior reference guide was used to avoid anterior femoral notching, and posterior condyles were the reference axis to set 
$3^{\circ}$ external rotation of the femoral component. For the tibia, the reference pod was attached to the extramedullary guide, and the leg was then moved into the abduction and adduction direction over the ankle center. The tibial resection was also performed perpendicularly to the MA in coronal plane and was set for $5^{\circ}$ of the posterior tibial slope. A validation tool was used to confirm both femoral and tibial cut orientation, and re-resection would be done if the cuts deviated $> \pm 1^{\circ}$ of the aiming target either in the coronal or sagittal plane. The patella was not resurfaced in both groups. The intramedullary opening of the femur was occluded with a bone plug in all knees of cBTKA group. A periarticular injection (PAI) mixture consisting of $100 \mathrm{mg}$ of Bupivacaine (0.5\% Marcaine; AstraZeneca, Sweden), $30 \mathrm{mg}$ of ketorolac tromethamine (ketorolac tromethamine $1 \mathrm{ml}$; SiuGuan, Taiwan), and sterile normal saline solution, was divided to be injected into bilateral knees. All patients in both groups received cemented, fixed-bearing, posterior stabilized prostheses (NexGen LPS, Zimmer Biomet, Warsaw, IN, USA). Before the arthrotomy closure, a suction drain was applied into the knee joint, and tranexamic acid $(15 \mathrm{mg} / \mathrm{kg})$ was poured into the joint. The drain was clamped for $3 \mathrm{~h}$, and a compressive dressing was applied following the arthrotomy closure. Afterward, the drain and dressing was removed at $24 \mathrm{~h}$ after the surgery.

Identical postoperative care was provided to all patients. For the first $48 \mathrm{~h}$, an intravenous patientcontrolled analgesia (PCA) morphine $(100 \mathrm{ml}$ solution containing $50 \mathrm{mg}$ of morphine sulphate) was injected as an on-demand bolus of $1 \mathrm{ml}$ with a $5 \mathrm{~min}$ lockout period, $30 \mathrm{mg}$ of ketorolac was given intravenously every $8 \mathrm{~h}$, and $500 \mathrm{mg}$ of oral acetaminophen was administered three times a day. Thereafter, all the catheters were discarded, and then $250 \mathrm{mg}$ of naproxen was given orally twice a day, as well as $500 \mathrm{mg}$ of acetaminophen was given orally three times a day. Two milligrams of morphine were additionally used for breakthrough pain every $4 \mathrm{~h}$ throughout hospitalization. Rehabilitation and a continuous passive motion (CPM) device were utilized for all patients to promote early ambulation with gait aids. All patients were administered with low molecular weight heparin for the first $48 \mathrm{~h}$, and combined with oral warfarin for 10 days.

The standard postoperative long-leg anteroposterior (AP) radiograph (digitalized) in weight bearing with fully extend knee and lateral weight-bearing knee film were obtained preoperative, and at 3-6 months of follow up. Patients were placed on a platform to visualize the ankle joint, while toes pointed forward and lateral malleoli was $20 \mathrm{~cm}$ apart. As a rotation control, the patella was placed perpendicular to the $\mathrm{x}$-ray beam. Only adequate radiograph without evidence of malrotation was analyzed. Four radiographic parameters were measured [14, 15]: (A) lower extremity MA; (B) coronal femoral component angle (CFA); (C) coronal tibial component angle (CTA); (D) sagittal femoral component angle (SFA); (E) sagittal tibial component angle (STA) or tibial slope. The coronal MA was determined by the line connecting the center of the femoral head and center of the ankle [14]. The MA is subdivided into the femoral MA, which is the line running from hip center through the center of intercondylar notch of the distal femur (or femoral prosthesis), and the tibial MA, which extends from the center of tibial plateau (or tibial tray) to the center of the talus $[14,15]$. A negative and positive value indicated a valgus and varus deformity, respectively. Postoperative CFA was defined as the angle formed by a line connecting the distal part of the medial and lateral condyles of the femoral component and the MA of femur. The angle that was formed by the MA of the tibia and a line parallel to the surface of the tibial tray, was determined as CTA. The angle $>90^{\circ}$ was defined as a valgus position, whereas the angle < $90^{\circ}$ represented a varus position. For the lateral weight-bearing knee film, the distal femoral and proximal tibial anatomical axes were set as the line connecting the midpoints of the outer cortex $5 \mathrm{~cm}$ and $15 \mathrm{~cm}$ proximal and distal to the joint line, respectively. The SFA and STA was defined as the angle formed by the line parallel to the distal femoral implant and the anatomical axis of femur, and a line parallel to the tibial baseplate and anatomical axis of tibia, respectively $[15,16]$. The angle $>90^{\circ}$ indicated an extension position, while the angle $<90^{\circ}$ was referred to a flexion position.

Outlier was defined as the alignment that deviating $> \pm 3^{\circ}$ from the satisfactory parameters which were $0^{\circ}$ of lower extremity $\mathrm{MA}, 90^{\circ}$ of CFA, $90^{\circ}$ of CTA, $90^{\circ}$ of SFA, and $85^{\circ}$ of STA (or $5^{\circ}$ of posterior tibial slope). The radiographs were evaluated by three assessors who were blind to the study group. All these parameters were compared between the two groups. Other outcomes including operative time, drain volume, hemoglobin $(\mathrm{Hb})$, calculated blood loss (CBL), allogeneic blood transfusion, intensity of pain determined by the $10-\mathrm{cm}$ visual analog scale (VAS), morphine consumption, maximal angle of knee flexion that the patient could tolerate as measure by the CPM device, and length of stay (LOS) that were prospectively collected at our institution, were also compared between groups.

The patient's total blood volume (TBV) was calculated using the equation of Nadler et al. [17]. The difference between preoperative and lowest postoperative $\mathrm{Hb}$ was applied with the hemoglobin balance method 
to determine CBL [18], which was accounted as the sum of external blood loss and hidden blood loss (extravasation into the soft tissues and loss due to hemolysis) [19].

$$
\begin{aligned}
\text { Male }: \operatorname{TBV}(\mathrm{ml})= & \left(0.0003669 \times \text { height }^{3}[\mathrm{~cm}]\right) \\
& +(32.19 \mathrm{x} \text { body weight }[\mathrm{kg}]) \\
& +604
\end{aligned}
$$

$$
\begin{aligned}
\text { Female }: \operatorname{TBV}(\mathrm{ml})= & \left(0.0003561 \times \text { height }^{3}[\mathrm{~cm}]\right) \\
& +(33.08 \times \text { body weight }[\mathrm{kg}]) \\
& +183
\end{aligned}
$$

Calculated blood loss $(\mathrm{ml})=\mathrm{TBV}[\mathrm{ml}] \mathrm{x}\left(\mathrm{Hb}_{\mathrm{i}}-\mathrm{Hb}_{\mathrm{e}}\right) / \mathrm{H}$ transfused $[\mathrm{ml}]$,

$\mathrm{Hb}_{\mathrm{i}}[\mathrm{g} / \mathrm{dl}]$ was defined as the preoperative $\mathrm{Hb}$, and $\mathrm{Hb}_{\mathrm{e}}[\mathrm{g} / \mathrm{dl}]$ was the lowest postoperative $\mathrm{Hb}$.

A serum $\mathrm{Hb}$ level below $9.0 \mathrm{~g} / \mathrm{dl}$ is rountinely indicated for a blood transfusion in BTKA at our institution. All of the clinical assessors were blinded to the treatment protocol.

\section{Statistical analysis}

All measured characteristics and outcomes were summarized with descriptive statistics. Data were checked with Kolmogorov - Smirnov tests for normality. Continuous data are presented as mean and standard deviation, and were compared between groups using the Student's $t$ test (normal distribution data) or the Mann-Whitney $U$ test (non-normally distributed data). Categorical data are presented as counts and percentages, and were compared by Fisher's exact test. Repeated measures ANOVA was also used to compare the time-dependent data including change of postoperative $\mathrm{Hb}, \mathrm{VAS}$, and knee flexion angle between groups. The post hoc comparisons of all pairwise of points in time were applied to account for multiple testing with Bonferroni adjustments. The sample size of iTKA and cTKA groups had $91.3 \%$ power to detect a difference of $200 \mathrm{~mL}$ in $\mathrm{CBL}$, which could significantly impact on blood transfusion rate, with standard deviation (SD) of $300 \mathrm{~mL}$ [20]; 84.8\% power to ascertain the minimal clinically important difference (MCID) of 1.5 for VAS with SD of 2.0 [21]; and 91.3\% power to detect $1^{\circ}$ difference of the mechanical axis with SD of $1.5^{\circ}$ [10], with type I error of $5 \%$. The inter- and intra-observer reliability based on the intraclass correlation coefficients (ICC) of the MA measurement were 0.842 and 0.918 , respectively. The inter- and intra-rater of ICC for tibial and femoral component alignment ranged from 0.746 to 0.818 . Statistical analysis was performed with the use of the Stata/MP 15.0 software (StataCorp LP, College Station, TX, USA). The level of significance was set at $P<0.05$.

\section{Results}

Comparing consecutive 44 iBTKA to 57 cBTKA procedures, no differences in the demographic data including age, gender, American Society of Anesthesiologists (ASA) physical status classification, Body mass index (BMI), and preoperative MA, were evident (Table 1). Preoperative $\mathrm{Hb}$ was comparable between the cBTKA and iBTKA groups. Postoperative $\mathrm{Hb}$ of the cBTKA group was significantly lower than those in the iBTKA group at $24 \mathrm{~h}(p=0.02)$, but this was not significantly different thereafter (Fig. 1). Operative duration, total drain volume, CBL, and blood transfusion rate were also not significantly different between the two groups

\section{Hb( Talglen2) of blood products}

There was no significant difference in postoperative VAS (Fig. 2a) and knee flexion angle between both groups (Fig. 2b). The cBTKA consumed total morphine of $14.61 \pm 9.96$ and the iBTKA group $14.30 \pm 9.84 \mathrm{mg}$ $(p=0.90)$ at $24 \mathrm{~h}$ postoperative, and of $23.01 \pm 16.79$ and $19.77 \pm 13.84 \mathrm{mg}$ respectively $(p=0.41)$ at $48 \mathrm{~h}$. The LOS of iBTKA was $5.43 \pm 1.54$ days, whereas it was $4.98 \pm$ 1.10 days for cBTKA group $(p=0.30)$.

For radiographic measures, the mean of MA and component orientation in the iBTKA group were more accurate than achieved in the cBTKA group $(p<0.01)$. The iBTKA group also had a significantly lower number of outliers that deviated $> \pm 3^{\circ}$ of neutral MA and aiming target of component positioning (except for SFA) (Table 3).

There were no intraoperative surgical complications identified in either group. For the cBKTA group, 1 patient experienced periprosthetic joint infection in unilateral knee, which was successfully treated with 2 staged revision TKA, and 1 had recurrent hemarthrosis in unilateral knee which was successfully treated with 1 staged revision TKA. One patient in the iBTKA group suddenly died at 4 months after full recovery from the surgery because of acute myocardial infarction.

\section{Discussion}

The clinical success of TKA basically depends on patient selection and surgical techniques used. Correct postoperative mechanical and component alignments are the paramount goal to guarantee optimal function and longevity of the implant [22]. In addition, a blood preserving strategy and multimodal pain control could also be additional keys for satisfactory TKA, and may be particularly important for BTKA because of its substantial bone and soft tissue trauma. Our study found comparable blood loss, and blood transfusion, between the iBTKA and cBTKA. As well, no differences between the $\mathrm{ABN}$ and conventional TKA regarding early postoperative VAS, morphine consumption, knee flexion angle, LOS, and complication rate, were found. 
Table 1 Demographic data and preoperative radiograph of the CBTKA and iBTKA groups

\begin{tabular}{|c|c|c|c|}
\hline & $\begin{array}{l}\text { CBTKA group } \\
(N=57)\end{array}$ & $\begin{array}{l}\text { iBTKA group } \\
(N=44)\end{array}$ & $p$ value \\
\hline No. of patients & 57 & 44 & \\
\hline Age (year) & $62.81 \pm 7.91$ & $64.89 \pm 6.20$ & 0.15 \\
\hline Gender (male/female) & $5 / 52$ & $5 / 39$ & 0.67 \\
\hline ASA (I/II/III) & $5 / 37 / 15$ & $0 / 31 / 13$ & 0.14 \\
\hline BMI $\left(\mathrm{kg} / \mathrm{m}^{2}\right)$ & $26.81 \pm 3.84$ & $26.79 \pm 4.28$ & 0.98 \\
\hline Preoperative mechanical axis (degree) & $5.02 \pm 1.09$ & $5.08 \pm 0.92$ & 0.66 \\
\hline Anatomical distal femoral angle (degree) & $97.43 \pm 2.01$ & $97.33 \pm 2.89$ & 0.74 \\
\hline Anatomical proximal tibial angle (degree) & $83.49 \pm 2.55$ & $84.10 \pm 3.25$ & 0.69 \\
\hline
\end{tabular}

Data are presented with mean \pm standard deviation (SD.) except for No. of patients, gender

For anatomical distal femoral and proximal tibial angle, the angle $>90^{\circ}$ was defined as a valgus position, whereas the angle $<90^{\circ}$ represented a varus position ASA American Society of Anesthesiologists (ASA) physical status classification, BMl body mass index, $\mathrm{kg} / \mathrm{m}^{2} \mathrm{kilogram} / \mathrm{meter}{ }^{2}$

Prior studies have revealed that $\mathrm{ABN}$ can reduce estimate blood loss and $\mathrm{Hb}$ dropping after surgery compared to conventional TKA [11, 12]. Ikawa et al. [10] conducted a prospective RCT study and reported that 121 unilateral ABN assisted TKA had $287 \mathrm{ml}$ less average blood loss than 120 unilateral conventional TKA $(p<0.05)$. However, comparable blood loss was proposed by other investigations [23, 24]. A recent systematic review and meta-analysis [8] also revealed an insignificant difference in postoperative blood lossrelated indicators between TKA using the ABN and the conventional system. Our results accorded with this as we found no difference between iBTKA and cBTKA in terms of CBL and blood transfusion rate, even if the postoperative $\mathrm{Hb}$ of cBTKA group dropped to a significantly lower level than the iBTKA group at $24 \mathrm{~h}$. While preclusion of intramedullary canal violation in iBTKA group might reduce drain volume by approximately 50 $\mathrm{ml}$, it seemed unable to reduce hidden blood loss even in a BTKA setting. Therefore, our finding suggested that using the conventional intramedullary guide might have no significant effect on total blood loss, when both the bone plug that occluded into the femoral opening hole, and the application of intra-articular tranexamic acid (TXA) used in our study may have been effective in mitigating blood loss after BTKA [20]. Additionally,

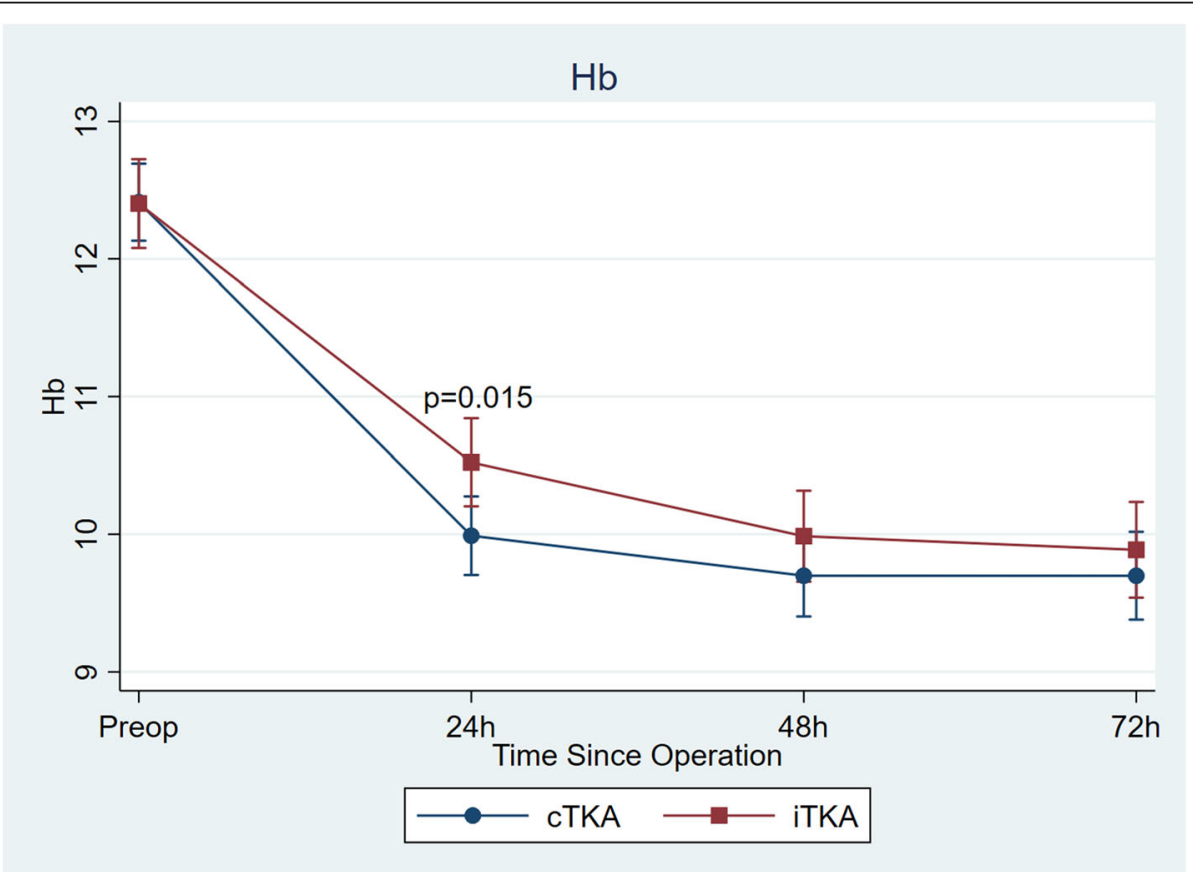

Fig. 1 Serum hemoglobin level obtained at preoperative, $24 \mathrm{~h}, 48 \mathrm{~h}$ and $72 \mathrm{~h}$ postoperative. Repeated measures ANOVA was used to compare between groups. Data are presented with mean $\pm 95 \%$ confidence interval. * $=$ statistically significant $(p=0.02)$ 
Table 2 The perioperative and postoperative characteristics of the CBTKA and iBTKA groups

\begin{tabular}{llll}
\hline & $\begin{array}{l}\text { CBTKA group } \\
(\boldsymbol{N}=\mathbf{5 7})\end{array}$ & $\begin{array}{l}\text { iBTKA group } \\
(\mathbf{N = 4 4 )}\end{array}$ & $\boldsymbol{p}$ value \\
\hline Operative duration $(\mathbf{m i n})$ & $127.25 \pm 18.95$ & $125.18 \pm 12.98$ & 0.54 \\
$\begin{array}{l}\text { Total drain volume } \\
(\mathbf{m l})\end{array}$ & $420.88 \pm 240.54$ & $354.55 \pm 176.43$ & 0.13 \\
CBL (ml) & $1052.41 \pm 343.87$ & $998.04 \pm 311.46$ & 0.42 \\
Blood transfusion & $43.86 \%$ & $43.18 \%$ & 0.95
\end{tabular}

Data are presented with mean \pm SD except blood transfusion rate min minutes, $\mathrm{m} /$ milliliters, $\mathrm{mg}$ milligrams, $C B L$ calculated blood loss

some studies have proposed that bleeding from trimmed cancellous bone of an uncovered resection surface [5, $25,26]$ and a soft tissue surface [3] may be more significant sources of bleeding after TKA.

It has also been suggested that intramedullary reaming and insertion of conventional instruments may inevitably lead to the destruction of endothelial cells, and dissipation of marrow emboli, which may subsequently induce a more inflammatory response and risk of embolismassociated morbidities such as acute cardiac disorders [7, 27, 28]. Accordingly, some inflammatory markers have been shown to be associated with functional recovery and morbidities following some major orthopedic surgeries [29-31]. However, our study demonstrated non-superiority of ABN over conventional TKA in regard to early postoperative pain, knee flexion, LOS, and complications.

For lower limb alignment, meta-analysis demonstrated that $A B N$ system significantly improved the accuracy of postoperative MA, and resulted in fewer number of outliers compared to the conventional system [8]. Additionally, the alignment of $\mathrm{ABN}$ assisted TKA was not inferior to computer assisted TKA (CAS), and it did not
Table 3 The postoperative radiographic outcomes of the CTKA and iTKA groups

\begin{tabular}{llll}
\hline Variables & $\begin{array}{l}\text { CTKA group } \\
(\boldsymbol{N}=\mathbf{5 7})\end{array}$ & $\begin{array}{l}\text { iTKa group } \\
(\boldsymbol{N}=\mathbf{4 4})\end{array}$ & $\boldsymbol{p}$ value \\
\hline Mechanical axis & $2.29^{\circ} \pm 0.81$ & $1.79^{\circ} \pm 0.85$ & $<0.01^{*}$ \\
MA outlier & $21.93 \%$ & $7.95 \%$ & $0.01^{*}$ \\
CFA & $91.74^{\circ} \pm 1.99$ & $90.60^{\circ} \pm 1.98$ & $<0.01^{*}$ \\
CFA outlier & $27.19 \%$ & $11.36 \%$ & $0.01^{*}$ \\
CTA & $87.77^{\circ} \pm 1.79$ & $89.18^{\circ} \pm 1.85$ & $<0.01^{*}$ \\
CTA outlier & $25.44 \%$ & $10.23 \%$ & $0.01^{*}$ \\
SFA & $89.28^{\circ} \pm 2.68$ & $90.54^{\circ} \pm 2.44$ & $<0.01^{*}$ \\
SFA outlier & $21.05 \%$ & $20.45 \%$ & $1.00^{*}$ \\
STA & $83.40^{\circ} \pm 3.32$ & $85.39^{\circ} \pm 2.60$ & $<0.01^{*}$ \\
STA outlier & $44.74 \%$ & $21.59 \%$ & $<0.01^{*}$ \\
\hline
\end{tabular}

Data are presented with mean \pm SD except all outliers

MA mechanical axis, CFA coronal femoral component angle, CTA coronal tibial component angle, SFA sagittal femoral component angle, STA sagittal tibial component angle or tibial slope

* = statistically significant $(p<0.05)$

increase risks associated with extra pin sites [9]. In our study, we found, if the ABN system was utilized, significantly better accuracy of MA and component positioning, and better reduction of outlier exceeding $\pm 3^{\circ}$ from the neutral mechanical alignment and aiming target of component orientation. Despite statistical significance, the mechanical alignment of the lower extremity that was improved by $0.5^{\circ}$ might still be questionable as to its clinical significance, particularly in regard to the longevity of the prostheses. Slevin et al. [32] assessed the whole leg coronal alignment and component position by using 3D-reconstructed computerized tomography scan (CT), and found that those parameters did not have significant correlation with patient outcomes after CAS TKA. Also, the benefit of CAS in terms of TKA survivorship could
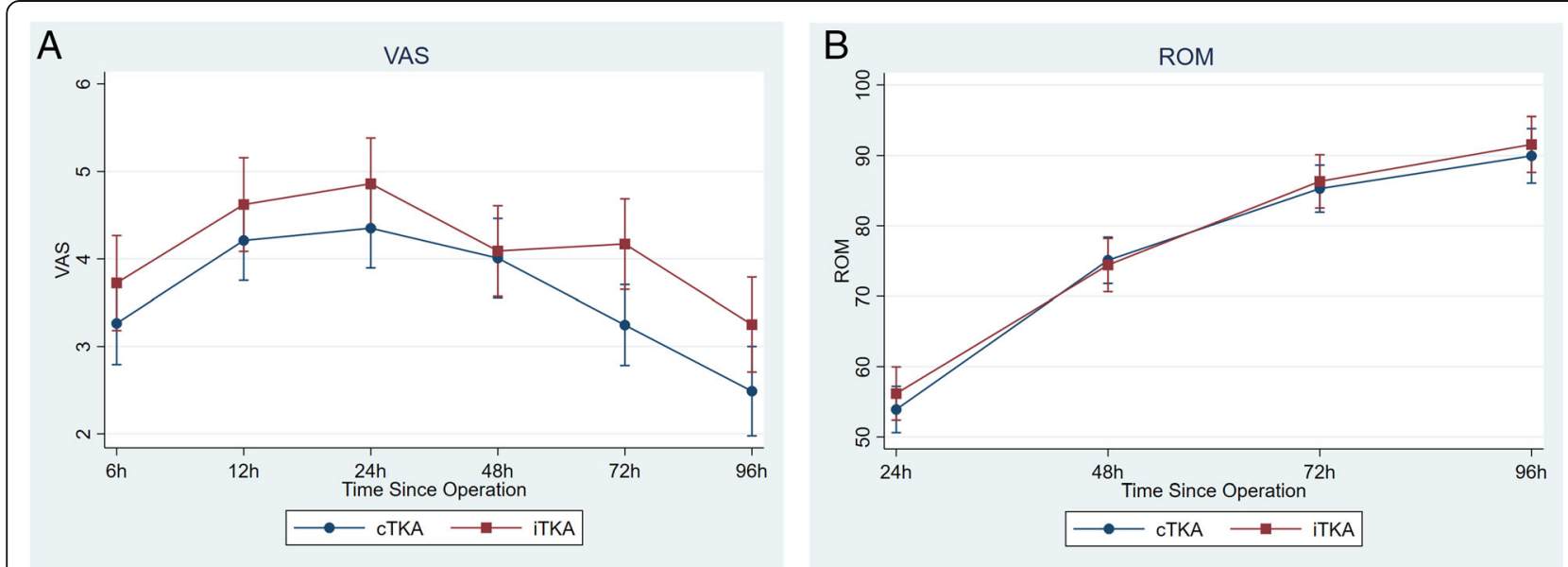

Fig. 2 a Visual analog scales (VAS) for pain intensity assessed at $6 \mathrm{~h}, 12 \mathrm{~h}, 24 \mathrm{~h}, 48 \mathrm{~h}$ and $72 \mathrm{~h}$ postoperative. b Knee flexion angle assessed at $24 \mathrm{~h}, 48 \mathrm{~h}$ and $72 \mathrm{~h}$ postoperative. Repeated measures ANOVA was used to compare between groups. Data are presented with mean $\pm 95 \%$ confidence interval 
not be proven even with long-term follow-up of between 14 and 16 years [33]. Similarly, ABN assisted TKA could not yield superiority in clinical outcomes over convention jig-assisted TKA as shown in recent systematic reviews and meta-analyses $[8,34]$. This may be due to the fact that $A B N$ does not significantly alter the surgical process used in conventional TKA, but it just provides accurate alignment information during the procedure. Recently, a systematic review by Budhiparama et al. [35] also found conflicting evidence as to whether ABN device decreases the number of outliers or improves the postoperative alignment to a clinically important level. On the other hand, using ABN might be associated with prolonged operative time [8] and additional cost of US\$850-\$1000 [34]. Research on cost-effectiveness of $\mathrm{ABN}$ systems may be in the early stages, and the result is still inconclusive.

It is acknowledged that our study has some limitations. First, this study is retrospective with some inherent limitations related to the study design. However, baseline characteristics were similar in of the iBTKA and cBTKA groups that were consecutively performed by a single surgeon with identical perioperative care. Second, the patients who enrolled in our study were predominantly female, which might be associated with poorer postoperative pain and less blood loss [36, 37]. Nevertheless, recent RCTs and systemic review could not identify gender as a significant predictor affecting blood loss, postoperative pain, and function recovery after TKA [26, 38, 39]. Third, use of PAI, non-steroidal anti-inflammatory drugs (NSAIDs), and topical TXA in our study protocol might be sufficient to reduce postoperative inflammation, pain, opioids consumption, and blood loss [20, 40], whereas different protocols might yield different findings. Lastly, $31.7 \%(32 / 101)$ of our patients had preoperative anemia which was defined as preoperative $\mathrm{Hb}$ $<12 \mathrm{~g} / \mathrm{dl}$ for female and $<13 \mathrm{~g} / \mathrm{dl}$ for male, and the blood transfusion threshold for BTKA at our institution is $9 \mathrm{~g} /$ dl. These might be an explanation for higher blood transfusion rate in the present study compared to previous reports.

\section{Conclusion}

ABN use in BTKA could not reduce blood loss, postoperative pain and complications, and did not improve functional recovery when compared to conventional BTKA. Although the ABN system significantly improved the accuracy of MA and prostheses positioning, and also reduced the number of outliers compared to conventional instruments, it is still controversial as to whether these improvements to lower limb alignment could benefit patients in regard to long-term functional outcomes and implant survival rate.

\section{Abbreviations}

TKA: Total knee arthroplasty; OA: Osteoarthritis; IM: Intramedullary;

EM: Extramedullary; CAS: Computer-assisted surgery; BTKA: Bilateral one-stage TKA; UTKA: Unilateral TKA; CBL: Calculated blood loss; TXA: Tranexamic acid; ABN: Accelerometer-based navigation; MA: Mechanical axis; CBTKA: Patients who had undergone BTKA with conventional instruments; iBTKA: Patients who had undergone BTKA with ABN; PAl: Periarticular injection; PCA: Patientcontrolled analgesia; CPM: Continuous passive motion; AP: Anteroposterior; CFA: Coronal femoral component angle; CTA: Coronal tibial component angle; SFA: Sagittal femoral component angle; STA: Sagittal tibial component angle; Hb: Hemoglobin; VAS: Visual analog scale; LOS: Length of stay;

TBV: Total blood volume; ICC: Interclass correlation coefficients;

ASA: American Society of Anesthesiologists; BMl: Body mass index;

$\mathrm{CT}$ : Computerized tomography

\section{Acknowledgements}

We thank Pariphat Chompoonutprapa, MD, and Parin Samapath, MD, for their technical assistance. Also, many thanks to Mr. Roy I. Morien of the Naresuan university Graduate School for his assistance in editing the English expression and grammar in this document.

\section{Authors' contributions}

AL contributed to the conception and design of the study, as well as surgeons who performed the operation. PR and AL wrote and completed the manuscript. PR, TT, WE and PT collected the required data. AL, PR and KP responsible for data interpretation and performed the statistical analysis. All authors have read and approved the manuscript.

\section{Funding}

This research did not receive any specific grant from funding agencies in the public, commercial, or not-for-profit sectors.

\section{Availability of data and materials}

The datasets used and/or analyzed during the current study are available from the corresponding author on reasonable request.

\section{Ethics approval and consent to participate}

The protocol of this study was approved by the Institutional Review Board (IRB) of Naresuan University (certificate of approval No.232/2018) and in accordance with the standards of the National Research Council. All patients provided written informed consent before participation.

\section{Consent for publication}

Not applicable.

\section{Competing interests}

The authors declare that they have no competing interests related to the study design, data collection, and results interpretation of this manuscript.

\section{Author details}

${ }^{1}$ Department of Orthopaedics, Faculty of Medicine, Naresuan University, 99 Moo 9 Thapho, Phitsanulok 65000, Thailand. ${ }^{2}$ Department of Preventive and Social Medicine, Faculty of Medicine, Chulalongkorn University, Bangkok, Thailand. ${ }^{3}$ Department of International Health, Johns Hopkins Bloomberg School of Public Health, Baltimore, MD, USA.

Received: 5 October 2020 Accepted: 12 January 2021

Published online: 10 February 2021

\section{References}

1. Odum SM, Troyer JL, Kelly MP, Dedini RD, Bozic KJ. A cost-utility analysis comparing the cost-effectiveness of simultaneous and staged bilateral Total knee Arthroplasty. J Bone Joint Surg Am. 2013;95:1441-9.

2. Bolognesi MP, Watters TS, Attarian DE, Wellman SS, Setoguchi S. Simultaneous vs staged bilateral total knee arthroplasty among Medicare beneficiaries, 2000-2009. J Arthroplast. 2013;28(8 Suppl):87-91.

3. Aggarwal AK, Shashikanth VS, Marwaha N. Platelet-rich plasma prevents blood loss and pain and enhances early functional outcome after total knee arthroplasty: a prospective randomised controlled study. Int Orthop. 2014; 38(2):387-95. https://doi.org/10.1007/s00264-013-2136-6 Epub 2013 Oct 11. PMID: 24114251; PMCID: PMC3923931. 
4. Liu W, Yang C, Huang X, Liu R. Tranexamic acid reduces occult blood loss, blood transfusion, and improves recovery of knee function after Total knee Arthroplasty: a comparative study. J Knee Surg. 2018;31(3):239-46.

5. Rattanaprichavej $P$, Laoruengthana A, Rasamimogkol S, Varakornpipat $P$, Reosanguanwong K, Pongpirul K. The effect of prosthesis design on blood loss in simultaneous bilateral Total knee Arthroplasty: closed-box versus open-box prosthesis. Clin Orthop Surg. 2019;11(4):409-15.

6. Han SB, Kim HJ, Kim TK, In Y, Oh KJ, Koh IJ, Lee DH. Computer navigation is effective in reducing blood loss but has no effect on transfusion requirement following primary total knee arthroplasty: a meta-analysis. Knee Surg Sports Traumatol Arthrosc. 2016;24(11):3474-81.

7. Kuo SJ, Wang FS, Wang CJ, Ko JY, Chen SH, Siu KK. Effects of Computer Navigation versus Conventional Total Knee Arthroplasty on Endothelial Damage Marker Levels: A Prospective Comparative Study. PLoS One. 2015; 10(5):e0126663.

8. Li JT, Gao X, Li X. Comparison of iASSIST navigation system with conventional techniques in Total knee Arthroplasty: a systematic review and meta-analysis of radiographic and clinical outcomes. Orthop Surg. 2019; 11(6):985-93.

9. Goh GS, Liow MH, Lim WS, Tay DK, Yeo SJ, Tan MH. Accelerometer-based navigation is as accurate as optical computer navigation in restoring the joint line and mechanical Axis after Total knee Arthroplasty: a prospective matched study. J Arthroplast. 2016;31(1):92-7.

10. Ikawa T, Takemura S, Kim M, Takaoka K, Minoda Y, Kadoya Y. Usefulness of an accelerometer-based portable navigation system in total knee arthroplasty. Bone Joint J. 2017;99-B(8):1047-52.

11. Xu X, Liu P, Yuan Z, Wang D, Lu Q, Zhang Z, Jiang Q, Shi D. Comparison of a novel handheld accelerometer-based navigation system and conventional instrument for performing distal femoral resection in total knee arthroplasty: a randomized controlled trial. Ann Transl Med. 2019;7(22):659.

12. Kawaguchi K, Michishita K, Manabe T, Akasaka Y, Higuchi J. Comparison of an accelerometer-based portable navigation system, patient-specific instrumentation, and conventional instrumentation for femoral alignment in Total knee Arthroplasty. Knee Surg Relat Res. 2017;29(4):269-75.

13. Chung BJ, Kang YG, Chang CB, Kim SJ, Kim TK. Differences between sagittal femoral mechanical and distal reference axes should be considered in navigated TKA. Clin Orthop Relat Res. 2009;467(9):2403-13. https://doi.org/ 10.1007/s11999-009-0762-5 Epub 2009 Feb 26.

14. Cherian JJ, Kapadia BH, Banerjee S, Jauregui JJ, Issa K, Mont MA. Mechanical, anatomical, and kinematic Axis in TKA: concepts and practical applications. Curr Rev Musculoskelet Med. 2014;7(2):89-95.

15. Gromov K, Korchi M, Thomsen MG, Husted H, Troelsen A. What is the optimal alignment of the tibial and femoral components in knee arthroplasty? Acta Orthop. 2014;85(5):480-7.

16. Lee S-S, Kim JH, Heo J-W, Moon Y-W. Gradual change in knee extension following total knee arthroplasty using ultracongruent inserts. Knee. 2019; 26(4):905-13. https://doi.org/10.1016/j.knee.2019.06.001 Epub 2019 Jun 19.

17. Nadler SB, Hidalgo $\mathrm{JH}$, Bloch T. Prediction of blood volume in normal human adults. Surgery. 1962;51(2):224-32.

18. Chen JY, Chin PL, Moo IH, Pang HN, Tay DK, Chia SL, et al. Intravenous versus intra-articular tranexamic acid in total knee arthroplasty: a double-blinded randomised controlled noninferiority trial. Knee. 2016; 23(1):152-6.

19. Sehat KR, Evans RL, Newman JH. Hidden blood loss following hip and knee arthroplasty. Correct management of blood loss should take hidden loss into account. J Bone Joint Surg Br. 2004;86(4):561-5.

20. Laoruengthana A, Rattanaprichavej P, Rasamimongkol S, Galassi M, Weerakul S, Pongpirul K. Intra-articular Tranexamic acid mitigates blood loss and morphine use after Total knee Arthroplasty. A Randomized Controlled Trial. J Arthroplast. 2019;34(5):877-81.

21. Tashjian RZ, Deloach J, Porucznik CA, Powell AP. Minimal clinically important differences (MCID) and patient acceptable symptomatic state (PASS) for visual analog scales (VAS) measuring pain in patients treated for rotator cuff disease. J Shoulder Elb Surg. 2009;18(6):927-32.

22. Ritter MA, Davis KE, Meding JB, Pierson JL, Berend ME, Malinzak RA. The effect of alignment and BMI on failure of total knee replacement. J Bone Joint Surg Am. 2011;93(17):1588-96.

23. Ueyama H, Minoda $Y$, Sugama R, Ohta Y, Yamamura K, Nakamura S, Takemura S, Nakamura H. An accelerometer-based portable navigation system improved prosthetic alignment after total knee arthroplasty in 3D measurements. Knee Surg Sports Traumatol Arthrosc. 2019;27(5):1580-6.
24. Moo IH, Chen JYQ, Chau DHH, Tan SW, Lau ACK, Teo YS. Similar radiological results with accelerometer-based navigation versus conventional technique in total knee arthroplasty. J Orthop Surg (Hong Kong). 2018;26(2):1-7.

25. Laoruengthana A, Rattanaprichavej $P$, Chaibhuddanugul N, Varakornpipat $P$, Galassi M, Pongpirul K. Blood loss reduction: effect of different knee prosthesis designs and use of tranexamic acid-a randomized controlled trial. Eur J Orthop Surg Traumatol. 2019;29(7):1519-24.

26. Moo IH, Chen JYQ, Pagkaliwaga EH, Tan SW, Poon KB. Bone wax is effective in reducing blood loss after Total knee Arthroplasty. J Arthroplast. 2017; 32(5):1483-7.

27. Kuo SJ, Hsu HC, Wang CJ, Siu KK, Hsu YH, Ko JY, Tang CH. Effects of computer-assisted navigation versus conventional total knee arthroplasty on the levels of inflammation markers: A prospective study. PLoS One. 2018; 13(5):e0197097.

28. Kalairajah Y, Cossey AJ, Verrall GM, Ludbrook G, Spriggins AJ. Are systemic emboli reduced in computer-assisted knee surgery?: a prospective, randomised, clinical trial. J Bone Joint Surg Br. 2006;88(2):198-202.

29. Sun T, Wang X, Liu Z, Chen X, Zhang J. Plasma concentrations of pro- and anti-inflammatory cytokines and outcome prediction in elderly hip fracture patients. Injury. 2011;42(7):707-13.

30. Hall GM, Peerbhoy D, Shenkin A, Parker CJ, Salmon P. Relationship of the functional recovery after hip arthroplasty to the neuroendocrine and inflammatory responses. Br J Anaesth. 2001;87(4):537-42.

31. Langkilde A, Jakobsen TL, Bandholm TQ, Eugen-Olsen J, Blauenfeldt T, Petersen J, et al. Inflammationand post-operative recovery in patients undergoing total knee arthroplasty-secondary analysis of a randomized controlled trial. Osteoarthr Cartil. 2017;25(8):1265-73.

32. Slevin O, Amsler F, Hirschmann MT. No correlation between coronal alignment of total knee arthroplasty and clinical outcomes: a prospective clinical study using 3D-CT. Knee Surg Sports Traumatol Arthrosc. 2017;25(12):3892-900. https://doi.org/10.1007/s00167-016-4400-y Epub 2016 Dec 22.

33. Kim YH, Park JW, Kim JS. 2017 Chitranjan S. Ranawat award: does computer navigation in knee Arthroplasty improve functional outcomes in young patients? A randomized study. Clin Orthop Relat Res. 2018;476(1):6-15.

34. Sun H, Li S, Wang K, Wu G, Zhou J, Sun X. Efficacy of portable accelerometer-based navigation devices versus conventional guides in Total knee Arthroplasty: a meta-analysis. J Knee Surg. 2020;33(7):691-703.

35. Budhiparama NC, Lumban-Gaol I, Ifran NN, Parratte S, Nelissen R. Does accelerometer-based navigation have any clinical benefit compared with conventional TKA? A systematic review. Clin Orthop Relat Res. 2019;477(9): 2017-29.

36. Barrington JW, Lovald ST, Ong KL, Watson HN, Emerson RH Jr. Postoperative Pain After Primary Total Knee Arthroplasty: Comparison of Local Injection Analgesic Cocktails and the Role of Demographic and Surgical Factors. J Arthroplast. 2016;31(9 Suppl):288-92.

37. Prasad N, Padmanabhan V, Mullaji A. Blood loss in total knee arthroplasty: an analysis of risk factors. Int Orthop. 2007;31(1):39-44.

38. Laoruengthana A, Jarusriwanna A, Rattanaprichavej P, Rasamimongkol S, Varakornpipat $P$, Pongpirul K. Timing of periarticular injection has no effect on postoperative pain and functional recovery in simultaneous bilateral total knee arthroplasty: a prospective randomized, double-blinded trial. BMC Musculoskelet Disord. 2019;20(1):162.

39. Alattas SA, Smith T, Bhatti M, Wilson-Nunn D, Donell S. Greater preoperative anxiety, pain and poorer function predict a worse outcome of a total knee arthroplasty. Knee Surg Sports Traumatol Arthrosc. 2017;25(11): 3403-10. https://doi.org/10.1007/s00167-016-4314-8 Epub 2016 Oct 12.

40. Kelley TC, Adams MJ, Mulliken BD, Dalury DF. Efficacy of multimodal perioperative analgesia protocol with periarticular medication injection in total knee arthroplasty: a randomized, double-blinded study. J Arthroplast. 2013;28(8):1274-7

\section{Publisher's Note}

Springer Nature remains neutral with regard to jurisdictional claims in published maps and institutional affiliations. 